$\Rightarrow$ MECHANISMS OF DISEASE

\title{
How stress affects fat
}

Although the mechanism by which obesity triggers insulin resistance is not fully understood, recent evidence suggests that inflammation in adipose tissue - triggered by macrophages that have infiltrated this tissue - contributes to this process. Roger Davis and colleagues now reveal that an inflammatory response within adipocytes regulates insulin sensitivity in the liver.

Various stresses activate the protein kinase Jun $\mathrm{N}$-terminal kinase 1 (JNK1). Feeding mice a high-fat diet, which induces obesity and insulin

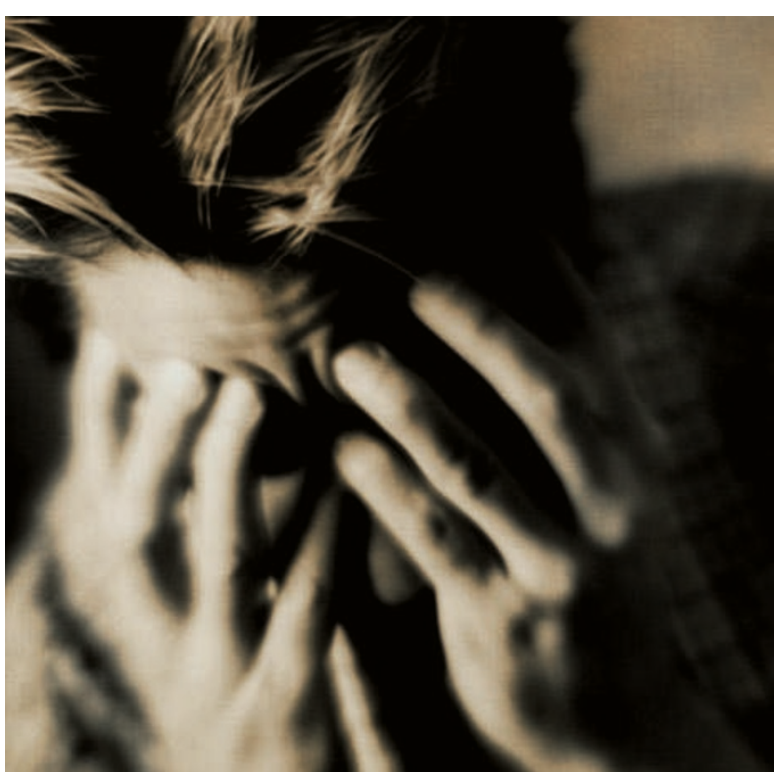

resistance, activates JNK1 in various organs, including the liver, adipose tissue and skeletal muscle. JNK1deficient mice do not develop insulin resistance, implicating this enzyme in the pathogenesis of insulin resistance. To determine whether a specific organ is responsible for JNK1mediated insulin resistance, Davis and colleagues generated mice with JNK1-deficient adipocytes and found that their susceptibility to insulin resistance induced by a high-fat diet was greatly reduced.

The authors then investigated various pro- and anti-inflammatory cytokines, as well as adipocytederived hormones that are thought to regulate insulin sensitivity, and found that only the abundance of interleukin 6 (IL-6) was substantially affected in mice with adipocyte-specific deficiency of JNK1. Increases in the serum concentration of IL- 6 and in the production of this cytokine in adipose tissue that are induced by a high-fat diet were almost completely eliminated by the ablation of JNK1 in adipocytes. Expression of SOCS3 - a signalling molecule that is activated by IL- 6 and inhibits insulin signalling - in the liver increased when wild-type mice were fed a high-fat diet; this effect was prevented by JNK1 ablation in adipocytes. So, IL-6-induced SOCS3 expression contributes to the development of hepatic insulin resistance.

This study underscores the prominent role of IL- 6 among adipose-tissue-secreted cytokines in the JNK1-mediated pathogenesis of liver insulin resistance. The authors propose a model in which cellular stress induced by a high-fat diet activates JNK1 in adipocytes, which results in an increased concentration of circulating IL-6. In the liver, IL-6 inhibits the action of insulin, probably through the induction of SOCS3 expression. JNK1 in adipocytes might contribute to activating macrophages in adipose tissue. Inflammatory cytokines secreted from activated macrophages probably augment the stress signal in adipocytes. Next, it will be interesting to investigate the source of IL-6; is it secreted from myeloid cells or adipocytes?

Ekat Kritikou

ORIGINAL RESEARCH PAPER Sabio, G. et al. A stress signaling pathway in adipose tissue regulates hepatic insulin resistance. Science 322 , 1539-1543 (2008) 